\title{
Photosynthesis of a Photocatalyst: Single Atom Pt Captured and Stabilized by Fe(III) Engineered Defect
}

Authors: Nicholas Anderson, ${ }^{\dagger}$ Tongzhou Xu, $\dagger^{\S}$ Mengyao Ouyang, ${ }^{\sharp}$ Patrick J. Bisson, ${ }^{\ddagger}$ Rebecca Davies, ${ }^{\ddagger}$ Joam Marmolejos, ${ }^{\star}$ and Mary Jane Shultz**

\section{SUPPORTING INFORMATION}

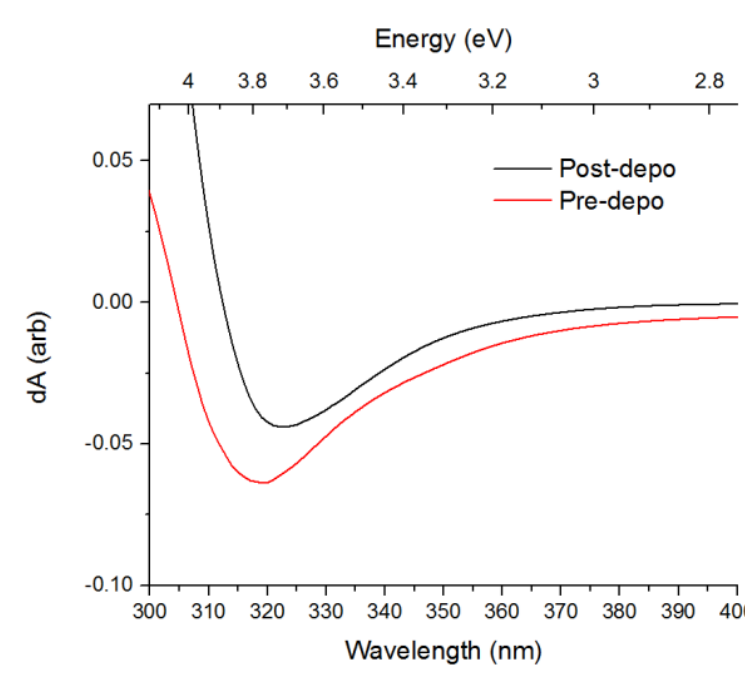

Figure S1: Nanoparticle size obtained from UV-Vis. First derivative UV-Vis spectra of Fe-TiUNP (red) and postphotodeposition $14.2 \% \mathrm{Pt}-\mathrm{Fe} \cdot \mathrm{TiUNP} / 85.8 \% \quad \mathrm{Fe}_{1} \cdot \mathrm{TiUNP}$ (black). Fe-TiUNP has a $318 \mathrm{~nm}$ band gap corresponding to a diameter of $1.61 \mathrm{~nm}$. $14.2 \% \mathrm{Pt}_{1}-\mathrm{Fe}_{1} \cdot \mathrm{TiUNP} / 85.8 \%$ $\mathrm{Fe}_{1} \cdot$ TiUNP has a $324 \mathrm{~nm}$ band gap. Observed red shift is consistent with deposition of Pt.

Particle size is determined via an empirical connection between the inflection point of the UV-Vis absorption and size as determined by SEM. ${ }^{53}$ Data for Fe-TiUNP and PtFe-TiUNP is shown in Figure S1. Deposition of Pt slightly red-shifts the minimum and broadens the peak. Taken together, these reflect Pt capture by a portion of the particles. The mix of smaller and larger particles results in the larger size distribution reflected in the peak width.

Photo-oxidation kinetics Impact of materials on photoefficiency is characterized via kinetics: The photocell is loaded with a solution containing the catalyst of interest, an initial concentration to target (formaldehyde in this case). The cell is irradiated with the lamp. Concentrations are chosen so that the target concentration is much greater than the catalyst particle concentration; the photon flux is smaller - the reaction is photon limited. Hence, the rate of target concentration reduction directly depends on the photoefficiency.

The impact of $\mathrm{Pt}$ is shown in Figure $\mathrm{S} 2$ and the main text, Figure 2. In the absence of Pt, formaldehyde is degraded at a rate of only $2.5 \% \mathrm{hr}^{-1}$. As controls, free $\mathrm{Pt}$ in solution produces only modest efficiency: only $5.5 \% \mathrm{hr}^{-1}$. In contrast, a mixture of $17 \%$ Pt-Fe-TiUNP and $83 \%$ Fe-TiUNP degrades $30 \% \mathrm{hr}^{-1}$. Kinetic data therefore reveal a cooperative effect between $\mathrm{Pt}$ and $\mathrm{Fe}$ which cannot be explained by simple addition of Pt precursor and Fe.TiUNP activities.

Photodeposition time characterization (Figure S3) To examine the impact of photodeposition time on $\mathrm{Pt}$ speciation and catalytic activity, $\mathrm{Pt}-\mathrm{Fe} \cdot \mathrm{TiO}_{2}$ materials were generated by photodeposition of Pt onto Fe.TiUNP. Single

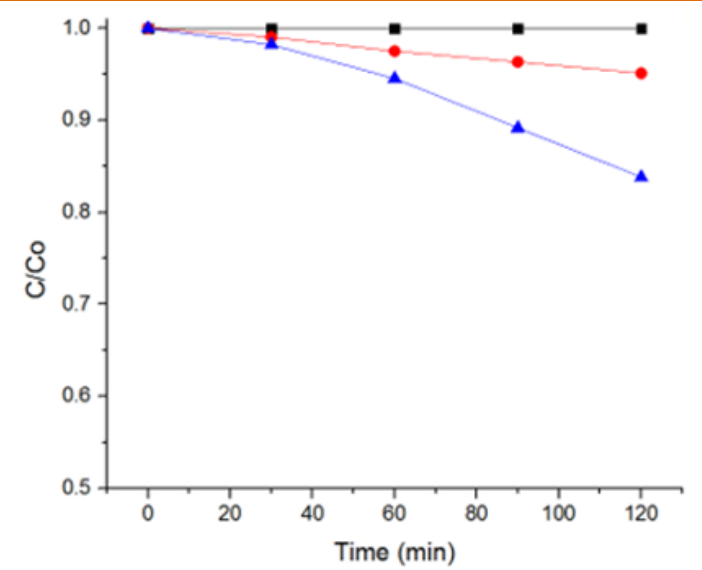

Figure S2: Effect of Pt loading on photocatalytic formaldehyde oxidation kinetics - controls and $\mathrm{Pt}$ deposition for <1 Fe: TiUNP: no catalyst (black), Fe.TiUNP without Pt (red), and Pt nanoparticles synthesized from irradiation of Pt precursor (blue). As shown in the main text, a mixture of SA Pt on Fe.TiUNP and undecorated Fe.TiUNP oxidizes $30 \% \mathrm{hr}^{-1}$.

\footnotetext{
Co-First authors

$\$$ Department of Chemistry, Laboratory for Water and Surface Studies, Pearson Lab, Tufts University, Medford, MA 02155 USA

$\S$ State Key Joint Laboratory of Environment Simulation and Pollution Control, School of Environment, Tsinghua University, Beijing 100084, China

\# Department of Chemical and Biochemical Engineering, Tufts University, Medford, MA 02155 USA

*Corresponding author email: Mary.Shultz@Tufts.edu
}

Submitted 
atom capture occurs for up to $4 \mathrm{hr}$ irradiation. Kinetic data shows that activity increases with increased Pt capture up to $4 \mathrm{hr}$.. Beyond $4 \mathrm{hr}$., Pt clusters are formed and activity decreases. At the $6 \mathrm{hr}$ mark the degradation rate drops to about a third that of a $4 \mathrm{hr}$ deposition.

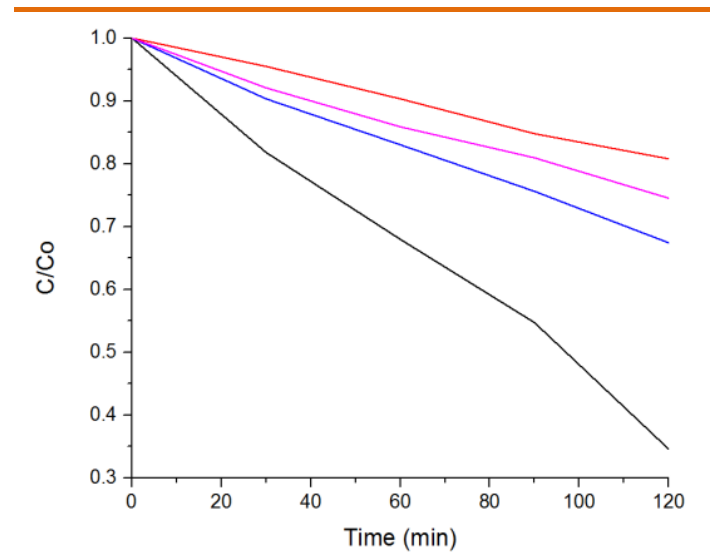

Figure S3: Effects of photodeposition time on photocatalytic activity. Initial concentration corresponding to $0.372 \mathrm{Pt}$ per TiUNP was photo deposited for varying times onto $0.4 \% \mathrm{Fe}_{1}$ TiUNP, then used in photodegradation of formaldehyde $\left(C_{\mathrm{o}}=1.67\right.$ $\mathrm{mM}$ ). Single Pt atom capture increases from (red) $0 \mathrm{hr}$; (magenta) $2 \mathrm{hr}$; (black) $4 \mathrm{hr}$. Beyond $4 \mathrm{hr}$, Pt clusters form and photodegradation decreases (blue).

\section{XAS data}

The Pt near edge structure (Figure S5) shifts blue for positive oxidation states relative to metallic $\mathrm{Pt}$. The maximum is about the same for $\mathrm{Pt}(\mathrm{II})$ in $\mathrm{Pt}(\mathrm{acac})_{2}$ and $\mathrm{Pt}(\mathrm{IV})$ in $\mathrm{H}_{2} \mathrm{PtCl}_{6}$. Insensitivity to the II vs IV oxidation state is due to excitation from the $\mathrm{L}$ shell as opposed to the $\mathrm{K}$ shell used for lighter metals. Near the more electronegative oxygen atom, the edge shifts to the blue. These observations are important for interpreting the oxidation state in the Pt-Fe.TiUNP particles and changes with photo-oxidation discussed in the main text.

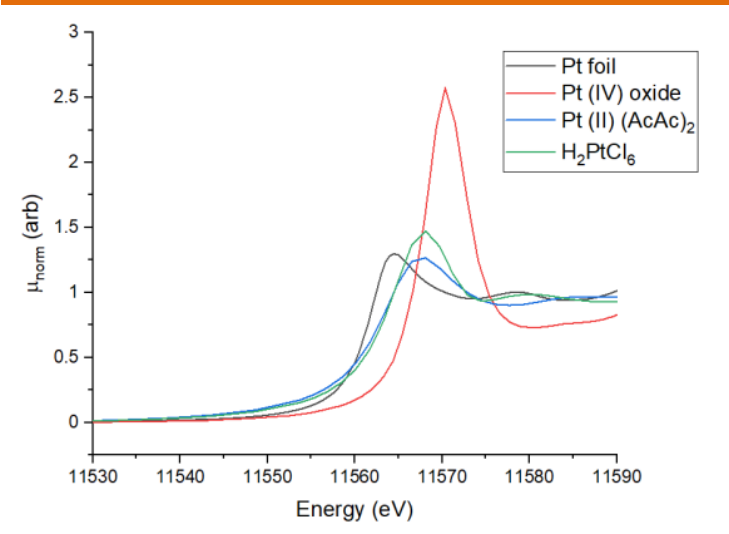

Figure S4: Pt XANES data for Pt (0), (II), and (IV) standards. The standard data indicate that differentiation between Pt (II) and Pt (IV) is quite difficult, as the $\mathrm{E}_{\mathrm{o}}$ values measured are almost identical.

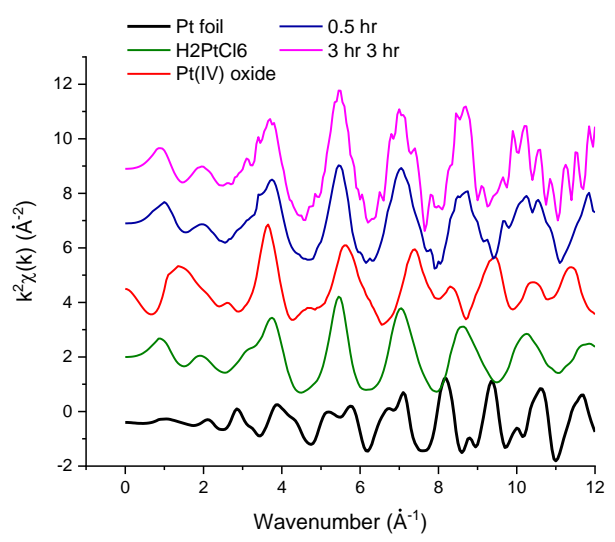

Figure S5: Pt foil, $\mathrm{H}_{2} \mathrm{PtCl}_{6}, \mathrm{Pt}(\mathrm{IV})$ oxide, and two deposition time $\mathrm{k}$-space plots for reference. Window from 2.5 to $16.5, \mathrm{k}$-weight $=2$. The more informative $I m$ spectra are contained in the body.

Oxide versus chloride bonding to $\mathrm{Pt}$ is shown by the $\mathrm{Im}$ part of the scattering spectrum, shown in the main text. Here (Figure S5) are shown the corresponding k-weight spectra. The greater noise in the deposition samples reflects the lower Pt content of the particles versus the foil and crystals. Scattering is a nonlinear process; the Im spectrum (shown in the main paper) is more informative since it is a linear combination of the scattering paths.

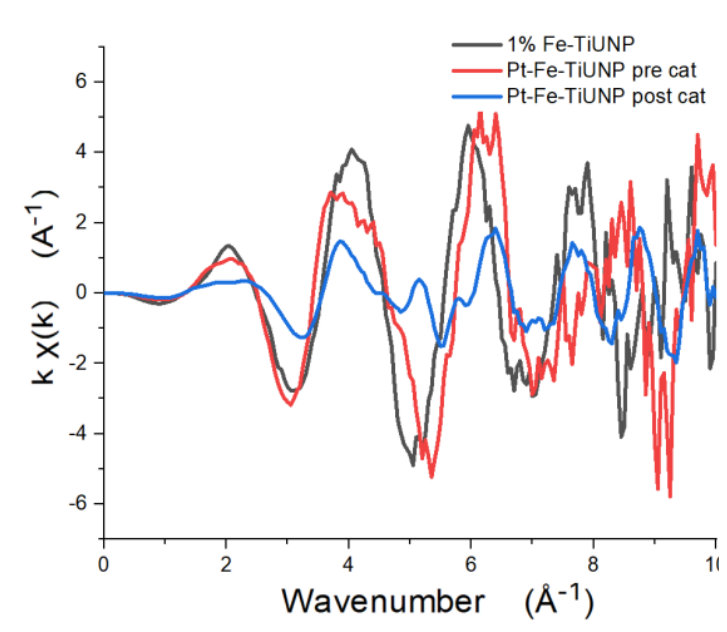

Figure S6: Fe-TiUNP and Pt-Fe-TiUNP k-space plots for reference. Window from $3-13$, k-weight $=1$.

\section{Comment on Polydispersity}

Discussion of polydispersity is beyond the scope of the current work. However, there is no evidence of large polydispersity in the particles; the accepted bulk $\mathrm{TiO}_{2}$ band gap is $385 \mathrm{~nm}$. Even small quantities of large particles in a sample result in a moderate rise in absorption at almost 60 $\mathrm{nm}$ before the measured band gap. The UV-VIS spectra of such samples show an increasing absorbance which begins 
at around $385 \mathrm{~nm}$ and continues to rise as the ultrasmall band gap is approached. This is not the case in the synthesized TiUNP UV-VIS spectrum: the rise in absorbance begins much bluer, somewhere around $340 \mathrm{~nm}$, and the background scattering of light from large $\mathrm{TiO}_{2} \mathrm{NPs}$ is not observed. Given the large index of refraction of $\mathrm{TiO}_{2}$, scattering can be observed for relatively small particles.

\section{Calculation 1:}

Photoefficiency is defined as the number of oxidations per photon absorbed. The number of photons is approximated as follows. The $\mathrm{Hg}$-Xe UV lamp outputs a light beam with a profile approximately equivalent to that in sunlight. The output that is absorbed by iron-doped titanium photocatalyst includes wavelengths from about $200 \mathrm{~nm}$ to $390 \mathrm{~nm}$. As a conservative estimate of the number of photons, approximate the photon energy as that at the bulk anatase band gap, $385 \mathrm{~nm}$.

The kinetic cell contains $25 \mathrm{~mL}$ a solution containing 50 $\mathrm{mg} / 30 \mathrm{~mL}$ titania photocatalyst. Under $1.5 \mathrm{~W}$ irradiation (typical kinetic run conditions), ultranano titanium dioxide dispersion absorbs $0.183 \mathrm{~W}$ more than does a control cell with 18 megaohm water. Past work indicates very little $(<0.1 \%)$ activity is due to visible light absorbance. The energy of a single photon is $E=h c / \lambda$, with $\lambda=385^{*} 10^{-9} \mathrm{~m}$ : $3.52 * 10^{17}$ photons/s: $1.29 * 10^{21}$ photons/hr.

In a typical kinetic run Fe-TiUNP solution degrades 1.25 $\mathrm{ppm}$ of formaldehyde $(2.5 \%$ of $50 \mathrm{ppm}$ initial formaldehyde) under the aforementioned $0.183 \mathrm{~W} \mathrm{UV}$ absorbance. A kinetic run degrades 1.04 micromolar formaldehyde. $((0.00125 \mathrm{~g} / \mathrm{L}) / 30 \mathrm{~g} / \mathrm{mol}) * .03 \mathrm{~L}$ of solution $* 6.023 * 10^{23}$ molecules/mol equals a total of $7.52 * 10^{18}$ molecules of formaldehyde degraded over the course of 60 minutes. Each formaldehyde requires the absorbance of 4 photons for its degradation: $\left(4 \times 7.52 * 10^{18}\right)$ molecules $/ \mathrm{hr}$ oxidations occur.

Putting these together, the photo efficiency $=\left(/ 1.29 * 10^{21}\right.$ photons $/$ hr $) * 4$ photons a molecule $=0.023 * 100 \%=2.3 \%$ for Fe-TiUNP. From the text, Pt-Fe-TiUNP is $15 \mathrm{x}$ as efficient as Fe.TiUNP, for a photo efficiency of $\sim 35 \%$. 